Artigo Original

Original Article

Valquíria Conceiçãa Souza ${ }^{1}$ Stela Maris Aguiar Lemos²

Descritores

Audição

Qualidade de Vida

Ambiente

Questionários

Pessoas com Deficiência

Keywords

Hearing

Quality of Life

Environment

Questionnaires

Disabled Persons
Endereço para correspondência:

Stela Maris Aguiar Lemos

Departamento de Fonoaudiologia,

Faculdade de Medicina, Universidade

Federal de Minas Gerais - UFMG

Av. Prof. Alfredo Balena, 190, Sala

251, Santa Efigênia, Belo Horizonte

(MG), Brasil, CEP: 30130-100.

E-mail: lemos.stela@gmail.com

\section{Restrição à participação de adultos e idosos: associação com fatores auditivos e socioambientais}

\author{
Participation restriction of adults and elderly \\ users of an audiology clinic: association with \\ auditory and social-environmental factors
}

\section{RESUMO}

Objetivo: Verificar a associação entre a restrição à participação auditiva com a qualidade de vida, a autopercepção de saúde, os fatores auditivos e os aspectos sociodemográficos de adultos e idosos atendidos em um serviço de audiologia. Método: Participaram do estudo 152 indivíduos e a restrição à participação auditiva foi avaliada por meio dos instrumentos Hearing Handicap Inventory for Adults - HHIA e o Hearing Handicap Inventory for Elderly - HHIE. Para a avaliação da qualidade de vida os participantes responderam o World Health Organization Quality of Life- versão abreviada (WHOQOL-bref). As características sociodemográficos foram avaliadas por meio de um questionário de caracterização dos participantes e pelo Critério Brasil ABEP, também foram coletados os resultados da avaliação auditiva. Foram realizadas análises estatísticas descritiva, bivariada $(p \leq 0,20)$ e regressão logística múltipla $(\mathrm{p} \leq 0,05)$ Resultados: Em relação à classe social, indivíduos pertencentes às classes $\mathrm{B} 1$ e $\mathrm{C} 2$ possuíam respectivamente, 4,75 e 7,73 vezes chances maiores de apresentar restrição à participação auditiva quando comparados aos indivíduos da classe D. Em relação aos fatores auditivos, ter perda auditiva incapacitante aumentou em 3,4 vezes a chance de apresentar percepção de restrição à participação auditiva. No domínio ambiental do instrumento Whoqol-Bref, a cada unidade aumentada no escore, houve diminuição de 0,96 vezes a chance de percepção de restrição na participação auditiva. Conclusão: Verificou-se que apenas o uso do aparelho de amplificação sonora individual, apesar dos benefícios, não foi capaz de eliminar a presença da restrição à participação auditiva da maioria dos participantes.

\begin{abstract}
Purpose: To verify the association between restrictions to auditory participation and quality of life, self-perceived health, auditory factors and sociodemographic aspects of adults and elderlies assisted in an audiology service. Methods: The study included 152 participants; restrictions to auditory participation were assessed using the instruments Hearing Handicap Inventory for Adults - HHIA and the Hearing Handicap Inventory for Elderly HHIE. In order to assess the quality of life, participants answered the World Health Organization Quality of Life - abbreviated version (WHOQOL-bref). Sociodemographic characteristics were assessed using a questionnaire to characterize the participants; and by the Brazilian Criteria ABEP. The results of the hearing assessment were also collected. Descriptive, bivariate statistical analyzes $(p \leq 0.20)$ and multiple logistic regression $(p \leq 0.05)$ were performed. Results: Regarding social class, individuals belonging to classes B1 and C2 had, respectively, 4.75 and 7.73 times greater chances of presenting restrictions to auditory participation compared to individuals of class D. Regarding hearing factors, disabling hearing loss increased by 3.4 times the chance of presenting perception of restriction to auditory participation. In the environmental domain of the WHOQOL-bref instrument, each unit increased in the score was associated with a decrease of 0.96 times in the chance of perceived restriction in auditory participation. Conclusion: We found that the use of the amplifying hearing aid by itself, despite its benefits, did not eliminate the restrictions on auditory participation of most participants.
\end{abstract}

Trabalho realizado na Universidade Federal de Minas Gerais - UFMG - Belo Horizonte (MG), Brasil

${ }^{1}$ Programa de Pós-graduação em Ciências Fonoaudiológicas, Universidade Federal de Minas Gerais - UFMG - Belo Horizonte (MG), Brasil.

${ }^{2}$ Departamento de Fonoaudiologia, Universidade Federal de Minas Gerais - UFMG - Belo Horizonte (MG), Brasil.

Fonte de financiamento: CNPq (Produtividade em Pesquisa - PQ 305782/2015-0), CAPES (Código de Financiamento 001).

Conflito de interesses: nada a declarar. 


\section{INTRODUÇÃO}

A perda auditiva é um importante problema de saúde pública e pode causar diversos impactos negativos. A Organização Mundial de Saúde (OMS), no ano 2018, estimou que aproximadamente 466 milhões de pessoas tinham perda auditiva incapacitante, ou seja, mais de $6 \%$ da população mundial, sendo que destes 432 milhões eram adultos. Além disso, projeções da OMS apontam que o número pode aumentar para 630 milhões em 2030 e ainda ser superior a 900 milhões em 2050 ${ }^{(1)}$. No Brasil, segundo o Instituto Brasileiro de Geografia e Estatística, no último censo demográfico, cerca de 9 milhões de cidadãos apresentavam perda auditiva, representando $5,1 \%$ da população e ainda de acordo com a mesma fonte, dentre as deficiências a auditiva foi a terceira mais relatada ${ }^{(2)}$.

A Organização Mundial de Saúde utiliza um modelo biossocial para caracterizar as consequências da perda auditiva na execução de funções (incapacidade) e também considera as adaptações ao meio ambiente e o envolvimento do indivíduo em situações do cotidiano (restrição à participação) ${ }^{(3)}$. Assim a restrição à participação auditiva está relacionada com a autopercepção do sujeito referente às limitações auditivas e aos possíveis impactos nas relações com familiares, amigos e estranhos, estilo de vida, situações sociais e emocionais e qualidade de vida ${ }^{(4)}$.

As consequências da perda auditiva variam entre sujeitos e são influenciadas pelas condições de saúde, nível de sociabilidade, capacidade de adaptação em situações adversas além das experiências ao longo da vida. Então, indivíduos com características auditivas semelhantes podem apresentar distintos níveis de restrição à participação e também não há associação direta entre a restrição e o grau da perda auditiva ${ }^{(4,5)}$.

Estudo realizado na Suécia com o objetivo de traduzir e validar o instrumento Amsterdam Inventory for Auditory Disability and Handicap (AIADH) e descrever as dificuldades auditivas a qualidade de vida dos 74 participantes, verificou que os homens apresentaram piores resultados na avaliação auditiva nas frequências de 2, 3,4 e $6 \mathrm{kHZ}$, piores escores na avaliação da localização da fonte sonora e utilizaram mais estratégias não verbais como forma de comunicação, porém apresentaram melhor qualidade de vida quando comparados às mulheres. Assim, os pesquisadores concluíram que apenas a avaliação auditiva não foi suficiente para determinar os efeitos psicossociais e na qualidade de vida nas pessoas portadoras de deficiência auditiva, também relataram a importância do desenvolvimento de programas de treinamento para a construção de ideias com objetivo de minimizar as consequências de uma deficiência auditiva ${ }^{()}$.

O uso do Aparelho de Amplificação Sonora Individual (AASI) é a intervenção primária para adultos com perda auditiva. Dentre os benefícios desse dispositivo, está a melhoria nas relações interpessoais, diminuição da incapacidade, das restrições à participação e redução da depressão ${ }^{(7,8)}$.

Estudo realizado no Reino Unido com objetivo de verificar a associação entre o uso de AASI e a melhora no desempenho cognitivo, este avaliado por meio do isolamento social e a presença depressão, avaliou 164, 770 indivíduos na faixa etária entre 40 a 69 anos. Nos resultados observouse que o uso do AASI melhorou o desempenho cognitivo independente de qualquer e associação positiva de uso do AASI e o isolamento social ou depressão. Diante dos resultados os autores concluíram que os efeitos do uso do AASI na cognição não está relacionado ao isolamento social ou depressão, os achados sugeriram que o benefício estava relacionado ao aumento da audibilidade de sons relacionados às atividades diárias dos participantes ${ }^{(9)}$.

Em outro estudo que investigou o nível de satisfação de 40 usuários de AASI, por meio da aplicação do Satisfaction Amplification in Daily Life (SADL), verificou-se que a participantes do estudo estavam satisfeitos com seus AASI durante a realização de atividades de vida diária, independentemente do tipo e grau da perda auditiva. Os autores também concluíram que os aconselhamentos e orientações adequadas influenciaram na avaliação positiva da satisfação ${ }^{(10)}$.

Os diversos impactos causados pela perda auditiva, não relacionados às alterações das funções e estruturas anatômicas, levaram os pesquisadores e profissionais a complementar as avaliações auditivas com o uso de questionários de referentes à restrição à participação auditiva e à qualidade de vida ${ }^{(11-14)}$. Esses instrumentos auxiliam na mensuração da eficácia e efetividade dos tratamentos.

Diante das informações apresentadas, o objetivo do presente estudo foi verificar a associação entre a restrição à participação auditiva com a qualidade de vida, os fatores auditivos e os aspectos sociodemográficos de adultos e idosos atendidos em um serviço de audiologia brasileiro.

\section{MÉTODO}

Trata-se de estudo observacional analítico transversal com amostra probabilística composta por 152 adultos e idosos atendidos no Setor de Audiologia de um hospital público na cidade de Belo Horizonte, estado de Minas Gerais.

A pesquisa foi aprovada pelo Comitê de Ética em Pesquisa da Universidade Federal de Minas Gerais, sob o parecer $\mathrm{n}^{\circ}$ CAAE 25014913.0.0000.5149. Todos os participantes da pesquisa foram informados sobre o caráter voluntário do estudo, seus objetivos e percursos metodológicos e concordaram em assinar o Termo de Consentimento Livre e Esclarecido (TCLE).

\section{Participantes}

Para a definição da casuística foi realizado cálculo amostral considerando-se o fluxo anual de 7680 indivíduos atendidos nos ambulatórios que constituem o Setor de Audiologia do hospital: Serviço de Saúde Auditiva e Ambulatório de Audiologia. Considerou-se uma amostragem aleatória simples e com nível de confiança de $99 \%$. Foi realizada estratificação da amostra por ambulatório de atendimento, participaram da pesquisa 114 indivíduos atendidos no Serviço de Saúde Auditiva e 38 do Ambulatório de Audiologia com idade igual ou superior a 18 anos. Os participantes realizaram os exames de audiometria tonal liminar e imitanciometria no dia da coleta de dados e assinaram o TCLE. 


\section{Restrição à participação auditiva}

Para a avaliação da autopercepção da restrição à participação auditiva foram utilizados os protocolos Hearing Handicap Inventory for Adults (HHIA) ${ }^{(15)}$ e o Hearing Handicap Inventory for Elderly (HHIE) ${ }^{(16)}$.

Ambos os questionários são formados por 25 questões e avaliam as consequências sociais e emocionais causados pela deficiência auditiva. Os valores da pontuação total poderiam variar de zero a 100 e quanto maior a pontuação maior foi a autopercepção da restrição à participação auditiva, ou seja, maiores foram as dificuldades auditivas e de aspectos não auditivos.

\section{Qualidade de vida}

A qualidade de vida dos participantes do presente estudo foi avaliada por meio da aplicação da versão validada para o português brasileiro do World Health Organization Quality of Life- versão abreviada (WHOQOL-bref) ${ }^{(17)}$. Este instrumento é composto por 26 questões, das quais duas referem-se à autopercepção da qualidade de vida e satisfação com a saúde. As outras 24 questões estão distribuídas em domínios. O instrumento foi aplicado em apenas um encontro e o aplicador solicitou ao entrevistado que para respondê-lo realizasse um resgate mnemônico da rotina das duas ultimas semanas, que antecederam a pesquisa, para escolher a resposta mais adequada à sua situação. As questões foram preenchidas com respostas que variaram a pontuação de 1 a 5 . A pontuação final de cada domínio foi calculada por uma sintaxe que considerou as respostas de cada questão, resultando em escores finais numa escala de 0 a 100, assim, quanto maior a pontuação melhor foi a avaliação apresentada pelo entrevistado ${ }^{(17)}$.

\section{Caracterização sociodemográfica e clínico-assistencial}

Para a obtenção dos dados sociodemográficos os participantes responderam um questionário de caracterização dos usuários dos ambulatórios e o Critério Brasil $\mathrm{ABEP}^{(18)}$. O questionário foi elaborado pelas pesquisadoras e apresentou perguntas referentes ao gênero, idade, local de moraria e escolaridade. Também foram coletadas informações referentes ao motivo e qual especialidade do profissional que realizou o encaminhamento para a avaliação auditiva. Os participantes da pesquisa realizaram autoavaliação da audição por meio de uma escala visual numerada de 0 a 10, quanto maior a pontuação melhor foi a avaliação.

Para complementar a avaliação dos dados sociodemográficos foi aplicado o Critério Brasil ABEP que é um instrumento que verifica as características domiciliares (presença e quantidade de itens na residência) e do nível de escolaridade do chefe de família. Para a análise do instrumento foram seguidos os critérios propostos pela literatura.

\section{Avaliação auditiva}

Para os resultados obtidos na audiometria tonal liminar e imitanciometria foram considerados os valores da melhor orelha. Os limiares audiométricos foram classificados quanto ao tipo e o grau. As perdas auditivas também foram classificadas como incapacitantes ou não incapacitantes. A Organização Mundial de Saúde define a perda auditiva incapacitante, em adultos, como a elevação permanente do limiar auditivo na melhor orelha para níveis acima de $40 \mathrm{dBNA}^{(19)}$.

\section{Análise estatística}

Foram realizadas análises descritivas das variáveis, por meio de distribuição de frequência absoluta e relativa das variáveis categóricas e de síntese numérica das variáveis contínuas.

Para a análise de associação foi selecionada como variável resposta restrição à participação auditiva e como variáveis explicativas qualidade de vida, aspectos sociodemográficos e auditivos. Para a avaliação da associação entre a variável resposta e as variáveis explicativas foi utilizado o teste Quiquadrado de Pearson para as variáveis categóricas e o teste não paramétrico de Man-Whitney para as variáveis contínuas, pois todas apresentaram distribuição assimétrica, avaliada pelo teste de Kolmogorov-Sminorv (valores de $\mathrm{p} \leq 0,05$ ). As variáveis com associação estatisticamente significantes ao nível de $20 \%$ $(\mathrm{p}<0,20)$ na análise univariada foram consideradas para entrada no modelo inicial de regressão logística múltipla. Foi adotado o método manual Backward, e foram mantidas as variáveis com associações ao nível de significância de 5\%. A magnitude das associações foi avaliada pelas Odds Ratio e seus respectivos intervalos de confiança a 95\%. A adequação dos modelos foi avaliada pelo teste de Hosmer e Lemeshow. Para todas as análises foi utilizado o programa Statistical Package for the Social Sciences (SPSS), versão 21.0.

\section{RESULTADOS}

A amostra do estudo foi composta por 152 indivíduos com idades entre 18 a 92 anos, a média etária foi de 61,8 anos. Participaram da pesquisa 90 idosos $(59,2 \%)$ e 62 adultos $(40,8 \%)$, verificou-se que a maioria dos sujeitos era do gênero feminino $(53,9 \%)$.

A maioria dos participantes $(63,2 \%)$ residia na cidade de Belo Horizonte, capital do estado Minas Gerais e $27 \%$ da amostra informou ter domicilio na região metropolitana da capital. Em relação à classe econômica, segundo critério proposto pelo o Critério Brasil ABEP, foram obtidos os seguintes resultados: classe A2 (0,7\%), classe B1 (14,5\%), classe B2 (12,5\%), classe C1 $(29,6 \%$, classe C2 $(26,3 \%)$ e classe D (16,4\%). Na avaliação da escolaridade, a média de anos de estudos dos participantes foi de 5,9 anos (mínimo 0 e máximo de 16 anos).

Em relação ao ambulatório de atendimento 75\% dos participantes foram atendidos no Serviço de Saúde Auditiva e 25\% no Ambulatório de Audiologia. A maioria dos encaminhamentos para a avaliação auditiva foi realizada por Otorrinolaringologistas (74,8\%), seguido pelos Fonoaudiólogos $(16,6 \%)$. A maioria dos participantes $(88,8 \%)$ já realizou avaliação auditiva anteriormente.

O resultado da avaliação da restrição à participação auditiva evidenciou que 63 participantes $(41,4 \%)$ não apresentaram restrição, 21 sujeitos $(13,8 \%)$ restrição leve, 20 (12,5\%) restrição 
moderada e 49 (32,2\%) indivíduos apresentaram restrição à participação auditiva significativa.

Os resultados apresentados a seguir referem-se à análise univariada de associação entre a restrição à participação auditiva com aspectos sóciodemográficos, fatores auditivos e qualidade de vida. Para a entrada no modelo inicial de regressão logística foram consideradas as associações ao nível de significância de $20 \%(\mathrm{p} \leq 0,20)$.

Dentre as variáveis analisadas, observou-se associação ao nível de $20 \%$ entre a presença de restrição à participação auditiva e o gênero feminino $(p=0,188)$ e com a classe econômica $(\mathrm{p}=0,036)$ conforme descrito na Tabela 1 .

$\mathrm{Na}$ análise de associação entre a presença de restrição à participação auditiva e os fatores auditivos verificou-se que todas as variáveis apresentaram significância estatística (Tabela 2). Pode-se destacar a associação positiva entre a presença de restrição à participação auditiva e as seguintes variáveis: presença de perda auditiva incapacitante $(\mathrm{p}=0,003)$ e autoavaliação da audição $(\mathrm{p}=<0,001)$.

Para a análise da associação entre a presença de restrição à participação auditiva e a qualidade de vida optou-se por

Tabela 1. Análise da associação entre a presença de restrição à participação auditiva e as variáveis sociodemográficas

\begin{tabular}{|c|c|c|c|}
\hline \multirow[t]{2}{*}{ Características } & \multicolumn{2}{|c|}{$\begin{array}{c}\text { Restrição à Participação } \\
\text { Auditiva }\end{array}$} & \multirow[t]{2}{*}{ Valor-p* } \\
\hline & Não & Sim & \\
\hline \multicolumn{4}{|l|}{ Gênero } \\
\hline Feminino & $30(47,6)$ & $52(58,4)$ & 0,188 \\
\hline Masculino & $33(52,4)$ & $37(41,6)$ & \\
\hline Total & $63(100,0)$ & $89(100,0)$ & \\
\hline \multicolumn{4}{|l|}{ Ciclo de vida } \\
\hline Adulto & $25(39,7)$ & $37(41,6)$ & 0,815 \\
\hline Idoso & $38(60,3)$ & $52(58,4)$ & \\
\hline Total & $63(100,0)$ & $89(100,0)$ & \\
\hline \multicolumn{4}{|l|}{ Escolaridade } \\
\hline$\leq 8$ anos & $43(68,3)$ & $63(72,4)$ & 0,581 \\
\hline$>8$ anos & $20(31,7)$ & $24(27,6)$ & \\
\hline Total & $63(100,0)$ & $87(100,0)$ & \\
\hline \multicolumn{4}{|l|}{ Critério Brasil ABEP } \\
\hline Classe B1 & $7(11,3)$ & $15(16,9)$ & 0,036 \\
\hline Classe B2 & $7(11,3)$ & $12(13,5)$ & \\
\hline Classe $\mathrm{C} 1$ & $24(38,7)$ & $21(23,6)$ & \\
\hline Classe C2 & $10(16,1)$ & $30(33,7)$ & \\
\hline Classe D & $14(22,6)$ & $11(12,4)$ & \\
\hline Total & $62(100,0)$ & $89(100,0)$ & \\
\hline \multicolumn{4}{|l|}{ Local de Residência } \\
\hline Belo Horizonte & $43(68,3)$ & $53(59,6)$ & 0,502 \\
\hline Região metropolitana & $15(23,8)$ & $26(29,2)$ & \\
\hline Outras & $5(7,9)$ & $10(11,2)$ & \\
\hline Total & $63(100,0)$ & $89(100,0)$ & \\
\hline \multicolumn{4}{|l|}{ Idade $_{\# \#}$} \\
\hline Mediana & 67,00 & 68,00 & $0,679^{\star \star}$ \\
\hline Média & 62,24 & 64,00 & \\
\hline Desvio Padrão & 18,21 & 17,26 & \\
\hline
\end{tabular}

reagrupar as categorias das questões referentes à autopercepção da qualidade de vida e da saúde (Tabela 3). Observou-se que todas as variáveis relacionadas à qualidade de vida tiveram associação com a presença da restrição à participação auditiva ao nível de $20 \%$. A média da pontuação de todos os domínios avaliados pelo Whoqol-bref foi menor para os indivíduos que apresentaram restrição à participação auditiva.

As análises do Odds Ratio (razão de chance) mostram que em relação à classe social, indivíduos pertencentes às classes $\mathrm{B} 1$ e $\mathrm{C} 2$ possuíam respectivamente, 4,75 e 7,73 vezes chances maiores de apresentar restrição na participação auditiva quando comparados com indivíduos da classe D (Tabela 4). Em relação aos fatores auditivos, ter perda auditiva incapacitante aumentou em 3,4 vezes a chance de apresentar percepção de restrição à participação auditiva. Também se verificou que quanto maior a nota da autoavaliação da audição menor foi a chance de ter percepção de restrição à participação auditiva. Quanto ao domínio ambiental do instrumento Whoqol-Bref, a cada unidade que se aumenta no escore desse domínio, diminui-se em 0,96 vezes a chance de percepção de restrição na participação auditiva.

Tabela 2. Análise da associação entre a presença de restrição à participação auditiva e fatores auditivos $(n=152)$

\begin{tabular}{|c|c|c|c|}
\hline \multirow{2}{*}{ Características } & \multicolumn{2}{|c|}{$\begin{array}{c}\text { Restrição à } \\
\text { Participação Auditiva }\end{array}$} & \multirow[t]{2}{*}{ Valor-p* } \\
\hline & Não & Sim & \\
\hline \multicolumn{4}{|l|}{ Ambulatório } \\
\hline Saúde Auditiva & $40(63,5)$ & $74(83,1)$ & 0,006 \\
\hline Audiologia & $23(36,5)$ & $15(16,9)$ & \\
\hline Total & $63(100,0)$ & $89(100,0)$ & \\
\hline \multicolumn{4}{|l|}{ Primeiro Exame } \\
\hline Sim & $13(20,6)$ & $4(4,5)$ & 0,002 \\
\hline Não & $50(79,4)$ & $85(95,5)$ & \\
\hline Total & $63(100,0)$ & $89(100,0)$ & \\
\hline \multicolumn{4}{|c|}{ Perda Auditiva Incapacitante } \\
\hline $\operatorname{Sim}$ & $31(49,2)$ & $65(73,0)$ & 0,003 \\
\hline Não & $32(50,8)$ & $24(27,0)$ & \\
\hline Total & $63(100,0)$ & $89(100,0)$ & \\
\hline \multicolumn{4}{|c|}{ Grau da Perda da Melhor Orelha } \\
\hline Normal & $21(33,3)$ & $13(14,6)$ & 0,009 \\
\hline Leve/Moderada & $33(52,4)$ & $50(56,2)$ & \\
\hline $\begin{array}{l}\text { Moderadamente Severa/ } \\
\text { Severa/Profunda }\end{array}$ & $9(14,3)$ & $26(29,2)$ & \\
\hline Total & $63(100,0)$ & $89(100,0)$ & \\
\hline \multicolumn{4}{|c|}{$\begin{array}{l}\text { Tipo da Perda Auditiva da Melhor } \\
\text { Orelha** }\end{array}$} \\
\hline Sem Perda Auditiva & $20(31,7)$ & $13(14,6)$ & 0,023 \\
\hline Neurossensorial & $36(57,1)$ & $57(64,0)$ & \\
\hline Condutiva/Mista & $7(11,1)$ & $19(21,3)$ & \\
\hline Total & $63(100,0)$ & $89(100,0)$ & \\
\hline \multicolumn{4}{|l|}{ Nota para a audição } \\
\hline Média & 7,2 & 5,5 & $<0,001$ \\
\hline Desvio padrão & 1,9 & 2,7 & \\
\hline Mediana & 7,0 & 5,0 & \\
\hline
\end{tabular}

*Qui-quadrado de Pearson; **Variável não considerada para a análise de Regressão Logística 
Tabela 3. Análise da associação entre a presença de restrição à participação auditiva e Qualidade de Vida (n=152)

\begin{tabular}{|c|c|c|c|}
\hline \multirow{2}{*}{ Características } & \multicolumn{2}{|c|}{ Restrição à Participação Auditiva } & \multirow{2}{*}{ Valor-p* } \\
\hline & Não & $\operatorname{Sim}$ & \\
\hline \multicolumn{4}{|l|}{ Qualidade de Vida } \\
\hline Boa/Muito Boa & $50(79,4)$ & $62(69,7)$ & \\
\hline Total & $63(100,0)$ & $89(100,0)$ & \\
\hline \multicolumn{4}{|l|}{ Satisfação com a Saúde } \\
\hline Satisfeito & $49(77,8)$ & $58(65,2)$ & \\
\hline Total & $63(100,0)$ & $89(100,0)$ & \\
\hline \multicolumn{4}{|l|}{ Domínio Físico } \\
\hline Média & 71,2 & 66,2 & 0,143 \\
\hline Desvio padrão & 17,9 & 20,5 & \\
\hline Desvio padrão & 19,7 & 20,6 & \\
\hline Mediana & 79,2 & 75,0 & \\
\hline \multicolumn{4}{|l|}{ Domínio Relações Sociais } \\
\hline Média & 77,6 & 71,8 & 0,118 \\
\hline Desvio padrão & 18,3 & 23,5 & \\
\hline Mediana & 75,0 & 75,0 & \\
\hline \multicolumn{4}{|l|}{ Domínio Ambiental } \\
\hline Média & 67,7 & 60,8 & 0,002 \\
\hline Desvio padrão & 12,9 & 14,7 & \\
\hline
\end{tabular}

*Teste Qui-quadrado de Pearson ou Teste de Mann Whitney

Tabela 4. Resultados da análise de regressão logística múltipla entre a presença de restrição à participação auditiva e variáveis selecionadas

\begin{tabular}{|c|c|c|c|c|}
\hline \multirow{2}{*}{ Características } & \multicolumn{2}{|c|}{ Modelo Inicial } & \multicolumn{2}{|c|}{ Modelo final } \\
\hline & OR (IC 95\%) & Valor-p* & OR (IC 95\%) & Valor-p* \\
\hline Sexo & $1,68(0,72-3,90)$ & 0,230 & $1,64(0,74-3,64)$ & 0,221 \\
\hline \multicolumn{5}{|l|}{ Critério Brasil ABEP } \\
\hline Classe B1 & $4,93(1,13-21,47)$ & 0,034 & $4,75(1,19-18,97)$ & 0,027 \\
\hline Classe B2 & $3,05(0,71-13,14)$ & 0,134 & $3,24(0,82-12,79)$ & 0,093 \\
\hline Classe $\mathrm{C} 1$ & $1,24(037,-4,18)$ & 0,724 & $1,25(0,40-3,88)$ & 0,703 \\
\hline Classe C2 & $9,21(2,20-38,53)$ & 0,002 & $7,73(2,07-28,90)$ & 0,002 \\
\hline Ambulatório & $1,84(0,57-5,97)$ & 0,308 & - & - \\
\hline Primeiro Exame & $2,69(0,62-11,74)$ & 0,188 & - & - \\
\hline Perda Auditiva Incapacitante & $2,96(0,84-10,43)$ & 0,092 & $3,40(1,44-8,04)$ & 0,005 \\
\hline \multicolumn{5}{|l|}{ Grau da Perda Auditiva } \\
\hline Leve/Moderada & $0,84(0,19-3,72)$ & 0,819 & - & - \\
\hline $\begin{array}{l}\text { Moderadamente Severa/Severa/ } \\
\text { Profunda }\end{array}$ & $1,11(0,17-7,13)$ & 0,912 & - & - \\
\hline Nota da Audição & $0,76(0,62-0,93)$ & 0,007 & $0,74(0,616-0,90)$ & 0,002 \\
\hline Qualidade de Vida & $0,94(0,32-2,82)$ & 0,917 & - & - \\
\hline Satisfação com a Saúde & $2,07(0,71-6,06)$ & 0,184 & - & - \\
\hline Domínio Físico & $1,01(0,98-1,04)$ & 0,580 & - & - \\
\hline Domínio Psicológico & $1,02(0,98-1,05)$ & 0,355 & - & - \\
\hline Domínio Relações Sociais & $1,03(0,98-1,03)$ & 0,800 & - & - \\
\hline Domínio Ambiental & $0,95(0,91-0,99)$ & 0,014 & $0,96(0,93-0,99)$ & 0,008 \\
\hline Constante & 1,30 & 0,897 & 22,16 & 0,011 \\
\hline
\end{tabular}

${ }^{*}$ Ajuste dos modelos inicial/final (Hosmer e Lemeshow): $p=0,728 / p=0,741$; Categorias de referência: Sexo - masculino; Critério Brasil ABEP- Classe D; Ambulatório

- audiologia; Primeiro exame - não; Perda incapacitante - não; Grau da Perda Auditiva - normal; Qualidade de Vida - ruim; Satisfação com a Saúde: insatisfeito 


\section{DISCUSSÃO}

A caracterização dos participantes da pesquisa mostrou o predomínio de idosos, mulheres e de usuários atendidos no Serviço de Saúde Auditiva. Esses aspectos podem ser explicados pela melhora nas condições de saúde e consequentemente aumento da expectativa de vida da população brasileira. Segundo informações do Instituto Brasileiro de Geografia e Estatística ${ }^{(2)}$, no ano 2000 os idosos representaram $7,1 \%$ da população, em 2013 essa representação passou a ser de 13\%. As mulheres também representam mais de $50 \%$ da população brasileira ${ }^{(2)}$.

O processo da perda auditiva decorrente do envelhecimento causa mudanças cognitivas, diminuição da compreensão de fala em ambientes ruidosos e impactos psicossociais ${ }^{(20)}$. Tanto na literatura brasileira ${ }^{(21,22)}$ quanto na européia ${ }^{(13,23)}$ e na norteamericana $^{(24)}$ estudos mostram os benefícios do uso do AASI para a diminuição dos impactos negativos na pessoa com deficiência auditiva. Diante da importância da promoção da saúde para a pessoa com deficiência auditiva, no ano 2004 o governo brasileiro instituiu a Política Nacional de Atenção à Saúde Auditiva, que oferta de forma gratuita o AASI e também realiza todo o acompanhamento desde a adaptação até a reabilitação auditiva ${ }^{(25)}$. O hospital escola onde a pesquisa foi desenvolvida é uma das referências no atendimento à pessoa com deficiência auditiva, situação que justifica a maior parte da amostra ser usuária do ambulatório do Serviço de Saúde Auditiva. Essas informações reforçam a importância das políticas públicas para a promoção do bem-estar e da tentativa de diminuir as possíveis restrições causadas pela perda auditiva.

$\mathrm{Na}$ associação entre a presença de restrição à participação auditiva e os aspectos sociodemográficos, tanto na análise univariada quanto na regressão logística, apenas a classificação socioeconômica apresentou resultado com significância estatística. Observou-se que ter baixa condição socioeconômica aumentou as chances do sujeito apresentar restrição á participação auditiva. O presente achado corrobora estudo ${ }^{(9)}$ que estimou a prevalência da deficiência auditiva em indivíduos na faixa etária entre 40 a 69 anos residentes no Reino Unido. Foram coletadas informações sobre o uso do Aparelho de Amplificação Sonora Individual (AASI), zumbido, exposição ao ruído e dados sociodemográficos. Os achados mostraram que $10 \%$ dos 164,770 participantes apresentavam deficiência auditiva e resultados com significância estatística entre a presença de deficiência auditiva e a condição socioeconômica inferior, idade avançada, além da exposição ao ruído no trabalho ou para escutar música alta.

Dentre as características da amostra também se verificou que a maior parte dos participantes apresentou baixa escolaridade, com menos de oito anos de estudo, porém não foi encontrada associação entre o nível de escolaridade e a presença da restrição a participação auditiva. A baixa escolaridade dificulta o indivíduo a conseguir oportunidades de emprego com melhor qualificação e que pagam salários mais altos, essas situações refletem na avaliação da condição socioeconômica. Contudo, houve associação entre a baixa condição socioeconômica e a presença de restrição à participação auditiva. Vale destacar que a escolaridade é um dos itens do Critério Brasil $\mathrm{ABEP}^{(18)}$, utilizado no presente estudo para analisar a condição socioeconômica. Deste modo, é possível inferir que os participantes com baixa escolaridade também apresentavam piores condições socioeconômicas.

Com relação ao nível de escolaridade, os achados da presente pesquisa não corroboram os resultados do estudo prospectivo realizado na Noruega com o objetivo de verificar a associação entre a perda auditiva e as condições socioeconômicas de 17.593 participantes. Os resultados evidenciaram que homens com menos de 10 anos de estudo e com ocupação profissional que requer menos anos de estudo têm chances maiores de apresentarem perda auditiva ${ }^{(5)}$. Em outro estudo de base populacional realizado na Suécia investigou-se a associação entre as dificuldades auditivas auto relatadas e as características sociodemográficas de 19.045 indivíduos na faixa etária de 20 a 64 anos. Os resultados indicaram que os participantes que obtinham a renda por meio da realização do trabalho manual, com menor nível de estudo, relataram maiores dificuldades auditivas quando comparados aos sujeitos que não realizavam trabalhos manuais ${ }^{(26)}$. A presente pesquisa por ter um delineamento transversal não verificou as causalidades dos resultados obtidos e o tamanho da amostra, menor quando comparado às pesquisas apresentadas, também pode ter influenciado nos achados.

Diante do exposto, pode-se inferir que os participantes da pesquisa brasileira por apresentarem nível de instrução educacional inferior e serem de baixa renda, ao longo da vida, ficaram mais vulneráveis a ocupações que exigiam menor qualificação acadêmica e também a ambientes de trabalho mais expostos ao ruído, situação que no decorrer dos anos aumentou o risco desses participantes adquirirem dificuldades auditivas que restringiram suas atividades e participação.

Segundo a literatura, um indivíduo com perda auditiva, ao procurar ajuda pode passar por estágios que envolvem o entendimento da perda auditiva, as experiências pessoais, interação com a sociedade e finalmente a decisão ${ }^{(27)}$. Nos resultados da presente pesquisa encontrou-se associação com significância estatística entre a presença de restrição à participação auditiva com a variável perda auditiva incapacitante e autoavaliação auditiva. O modelo de regressão logística Odds Ratio evidenciou que apresentar perda auditiva incapacitante e ter baixa pontuação na avaliação da audição aumenta as chances do indivíduo apresentar restrição à participação auditiva. Os achados da presente pesquisa corroboram estudo ${ }^{(27)}$ cujo objetivo foi investigar a aceitação da perda auditiva e mostrou que a não aceitação da perda auditiva estava relacionada ao aumento do desconforto emocional, situação que pode gerar restrição à participação auditiva.

$\mathrm{Na}$ associação entre a presença de restrição à participação auditiva e a qualidade de vida, observou-se na avaliação global que a maioria dos participantes da pesquisa relatou ter qualidade de vida boa ou muito boa e estavam satisfeitos com o estado geral de saúde. A avaliação da qualidade de vida é algo subjetivo e está relacionado com múltiplos fatores dentre eles a estabilidade das relações interpessoais e o bom estado de saúde ${ }^{(28)}$. A qualidade de vida também é influenciada pela capacidade do individuo adaptar-se ao meio no qual está inserido. Idosos com melhor capacidade de adaptação tendem a apresentar menores impactos emocionais diante das adversidades do cotidiano ${ }^{(29)}$. Diante dos resultados apresentados infere-se que os participantes da pesquisa associaram a avaliação positiva da qualidade de vida 
ao bom estado de saúde. Também pode-se considerar que os participantes, a maioria idosos, ao avaliarem a qualidade de vida estavam conscientes das possíveis limitações causadas pelo envelhecimento e se adaptaram à essa condição de vida.

Em relação aos quatro domínios avaliados pelo Whoqolbref, tanto na análise univariada quanto no modelo de regressão logística, verificou-se relação com significância estatística entre a presença de restrição a participação auditiva e o domínio ambiental. Este domínio apresenta questões referentes à segurança, ao ambiente físico, aos recursos financeiros, às oportunidades de adquirir informações, ao lazer e transportes. Esse achados corroboram estudo brasileiro que avaliou a qualidade de vida de idosos com audição normal e presbiacúsicos usuários de $\mathrm{AASI}^{(28)}$. Nessa pesquisa, que também utilizou o Whoqol-bref, observou-se associação positiva entre a presbiacusia e a pior percepção da qualidade de vida no domínio ambiental, assim os resultados indicaram que a perda auditiva, independente do uso do AASI, limita o acesso ao meio.

Os outros domínios avaliados pelo Whoqol-bref, que não apresentaram associação com significância estatística com a presença de restrição à participação, avaliaram os aspectos físicos, psicológicos e as relações sociais. Os achados da presente pesquisa corroboram em parte com uma pesquisa norte-americana ${ }^{(30)}$. $\mathrm{Na}$ avaliação da qualidade de vida, não foram observadas diferenças com significância estatística entre o uso de AASI e a otimização dos aspectos cognitivos e do comportamento social, porém observou-se relação com significância estatística entre o uso do AASI e a melhora dos aspectos físicos. Diferente dos achados do presente estudo, outra pesquisa brasileira ${ }^{(29)}$ cujo objetivo era avaliar a qualidade de vida de idosos antes e após a adaptação do AASI evidenciou melhora significativa na avaliação dos domínios físico, psicológico e relações sociais após a adaptação do AASI. Esses dados mostram a importância do encaminhamento para os serviços de adaptação de AASI e também para a reabilitação auditiva como auxilio para a melhora de aspectos emocionais e sociais em indivíduos com perda auditiva.

\section{CONCLUSÃO}

Os resultados do presente estudo mostraram que indivíduos com perda auditiva incapacitante e com condições socioeconômicas mais baixas têm maiores chances de apresentarem restrição à participação auditiva, os achados também evidenciam a importância de considerar a influência dos fatores ambientais no impacto da restrição. Na presente pesquisa verificou-se que apenas o uso do AASI, apesar dos benefícios, não foi capaz de eliminar a restrição à participação auditiva da maioria dos participantes, esses achados reforçam a importância de intervenções clínicas que considerem os resultados da avaliação auditiva e também o contexto de vida de cada paciente.

\section{REFERÊNCIAS}

1. WHO: World Health Organization. Addressing the rising prevalence of hearing loss [Internet]. Geneva: WHO; 2018 [citado em 2020 Jun 30]. Disponível em: https://apps.who.int/iris/bitstream/handle/10665/260336/9789241550260eng.pdf? sequence $=1 \& u a=1$

2. IBGE: Instituto Brasileiro de Geografia e Estatística. Censo demográfico: resultados [Internet]. Rio de Janeiro: IBGE; 2014 [citado em 2015 Nov 9]. Disponível em: http://censo2010.ibge.gov.br/resultados
3. Alfakir R, Holmes AE, Noreen F. Functional performance in older adults with hearing loss: application of the International Classification of Functioning brief core set for hearing loss: a pilot study. Int J Audiol. 2015;54(9):57986. http://dx.doi.org/10.3109/14992027.2015.1023903. PMid:25816864.

4. Helvik AS, Jacobsen G, Wennberg S, Arnesen H, Ringdahl A, Hallberg LR. Activity limitation and participation restriction in adults seeking hearing aid fitting and rehabilitation. Disabil Rehabil. 2006;28(5):281-8. http:// dx.doi.org/10.1080/09638280500160311. PMid:16492622.

5. Helvik AS, Krokstad S, Tambs K. Socioeconomic inequalities in hearing loss in a healthy population sample: the HUNT study. Am J Public Health. 2009;99(8):1376-8. http://dx.doi.org/10.2105/AJPH.2007.133215. PMid:19542048.

6. Hallberg LR, Hallberg U, Kramer SE. Self-reported hearing difficulties, communication strategies and psychological general well-being (quality of life) in patients with acquired hearing impairment. Disabil Rehabil. 2008;30(3):203-12. http://dx.doi.org/10.1080/09638280701228073. PMid:17852289.

7. McArdle R, Chisolm TH, Abrams HB, Wilson RH, Doyle PJ. The WHODAS II: measuring outcomes of hearing aid intervention for adults. Trends Amplif. 2005;9(3):127-43. http://dx.doi.org/10.1177/108471380500900304. PMid: 16244759

8. Dawes P, Cruickshanks KJ, Fischer ME, Klein BE, Klein R, Nondahl DM. Hearing-aid use and long-term health outcomes: hearing handicap, mental health, social engagement, cognitive function, physical health, and mortality. Int J Audiol. 2015;54(11):838-44. http://dx.doi.org/10.3109/14 992027.2015.1059503. PMid:26140300.

9. Dawes P, Fortnum H, Moore DR, Emsley R, Norman P, Cruickshanks $\mathrm{K}$, et al. Hearing in middle age: a population snapshot of 40 - to 69 -year olds in the United Kingdom. Ear Hear. 2014;35(3):e44-51. http://dx.doi org/10.1097/AUD.0000000000000010. PMid:24518430.

10. Dashti R, Khiavi FF, Sameni SJ, Bayat A. Satisfaction with hearing aids among aged patients with different degrees of hearing loss and length of daily use. J Audiol Otol. 2015;19(1):14-9. http://dx.doi.org/10.7874/ jao.2015.19.1.14. PMid:26185786.

11. Lutman ME, Robinson DW. Quantification of hearing disability for medicolegal purposes based on self-rating. Br J Audiol. 1992;26(5):297306. http://dx.doi.org/10.3109/03005369209076651. PMid:1486358.

12. Kramer SE, Kapteyn TS, Festen JM, Tobi H. Factors in subjective hearing disability. Audiology. 1995;34(6):311-20. http://dx.doi. org/10.3109/00206099509071921. PMid:8833311.

13. Metselaar M, Maat B, Krijnen P, Verschuure H, Dreschler WA, Feenstra L. Self-reported disability and handicap after hearing-aid fitting and benefit of hearing aids: comparison of fitting procedures, degree of hearing loss, experience with hearing aids and uni- and bilateral fittings. Eur Arch Otorhinolaryngol. 2009;266(6):907-17. http://dx.doi.org/10.1007/s00405008-0847-x. PMid:19005632.

14. Umansky AM, Jeffe DB, Lieu JE. The HEAR-QL: quality of life questionnaire for children with hearing loss. J Am Acad Audiol. 2011;22(10):644-53. http://dx.doi.org/10.3766/jaaa.22.10.3. PMid:22212764.

15. Newman CW, Weinstein BE, Jacobson GP, Hug GA. The Hearing Handicap Inventory for Adults: psychometric adequacy and audiometric correlates. Ear Hear. 1990;11(6):430-3. http://dx.doi.org/10.1097/00003446-19901200000004. PMid:2073976.

16. Ventry IM, Weinstein BE. The hearing handicap inventory for the elderly: a new tool. Ear Hear. 1982;3(3):128-34. http://dx.doi.org/10.1097/00003446198205000-00006. PMid:7095321.

17. leck MPA, Louzada S, Xavier M, Chachamovich E, Vieira G, Santos $\mathrm{L}$, et al. Application of the Portuguese version of the abbreviated instrument of quality life WHOQOL-bref. Rev Saude Publica. 2000;34(2):178-83. http://dx.doi.org/10.1590/S0034-89102000000200012. PMid:10881154.

18. ABEP: Associação Brasileira de Empresas de Pesquisa. Critério de Classificação Econômica Brasil [Internet]. São Paulo: ABEP; 2014 [citado em 2015 Out 9]. Disponível em: http://www.abep.org/criterio-brasil

19. WHO: World Health Organization. Deafness and hearing loss [Internet] Geneva: WHO; 2020 [citado em 2020 Jun 30]. Disponível em: https:// www.who.int/en/news-room/fact-sheets/detail/deafness-and-hearing-loss

20. Chisolm TH, Willott JF, Lister JJ. The aging auditory system: anatomic and physiologic changes and implications for rehabilitation. Int $\mathrm{J}$ Audiol. 2003;42(Supl. 2):S3-10. http://dx.doi.org/10.3109/14992020309074637. PMid:12918622. 
21. Luz VB, Silva MC, Scharlach RC, Iório MCM. Correlation between the handicap and the benefit of hearing aid use in adults and elderly. Rev Soc Bras Fonoaudiol. 2011;16(2):160-6. http://dx.doi.org/10.1590/S1516-80342011000200009.

22. Guarinello AC, Marcelos SB, Ribas A, Marques JM. Analysis of the perception of a group of seniors about their hearing handicap before and after hearing aid use. Rev Bras Geriatr Gerontol. 2013;16(4):739-45. http:// dx.doi.org/10.1590/S1809-98232013000400008.

23. Vuorialho A, Karinen P, Sorri M. Counselling of hearing aid users is highly cost-effective. Eur Arch Otorhinolaryngol. 2006;263(11):988-95. http:// dx.doi.org/10.1007/s00405-006-0104-0. PMid:16799805.

24. Preminger JE, Meeks S. Evaluation of an audiological rehabilitation program for spouses of people with hearing loss. J Am Acad Audiol. 2010;21(5):315-28. http://dx.doi.org/10.3766/jaaa.21.5.4. PMid:20569666.

25. Brasil. Ministério da Saúde. Portaria n. 2.073/GM, de 28 de setembro de 2004. Institui a Política Nacional de Atenção à Saúde [Internet]. Diário Oficial da União; Brasília; 2004 [citado em 2016 Jan 20]. Disponível em: http://bvsms. saude.gov.br/bvs/saudelegis/gm/2004/prt2073_28_09_2004.html

26. Pierre PV, Fridberger A, Wikman A, Alexanderson K. Self-reported hearing difficulties, main income sources, and socio-economic status; a cross-sectional population-based study in Sweden. BMC Public Health. 2012;12:874. http://dx.doi.org/10.1186/1471-2458-12-874. PMid:23067045.
27. Manchaiah VKC, Molander P, Rönnberg J, Andersson G, Lunner T, The acceptance of hearing disability among adults experiencing hearing difficulties: a cross-sectional study. BMJ Open. 2014;4(1):e004066. http:// dx.doi.org/10.1136/bmjopen-2013-004066. PMid:24401726.

28. Ribas A, Kozlowski L, Almeida G, Marques JM, Silvestre RAA, Mottecy CM. Quality of life: comparing results in elderly with and withou presbyacusis. Rev Bras Geriatr Gerontol. 2014;17(2):353-62. http://dx.doi. org/10.1590/S1809-98232014000200012.

29. Mondelli MF, Souza PJ. Quality of life in elderly adults before and after hearing aid fitting. Rev Bras Otorrinolaringol. 2012;78(3):49-56. PMid:22714847.

30. Dawes P, Emsley R, Cruickshanks KJ, Moore DR, Fortnum H, EdmondsonJones M, et al. Hearing loss and cognition: the role of hearing aids, social isolation and depression. PLoS One. 2015;10(3):e0119616. http://dx.doi. org/10.1371/journal.pone.0119616. PMid:25760329.

\section{Contribuição dos autores}

VCS participou da concepção do estudo, coleta e análise de dados, redação do manuscrito e aprovação da versão final; SMAL participou, na condição de orientadora, da concepção do estudo, análise e interpretação dos dados, redação do manuscrito e aprovação da versão final. 